\title{
Fälle-für-Alle: Auf dem Weg zu einer konstruktiven Fehlerkultur
}

Martina König-Bachmann, Christian Ederer, Innsbruck; Isabella Romano, Bern; Regine Knobloch, Karlsruhe; Ans Luyben, Thun; Patricia Gruber, Wetter; Christiane Schwarz, Hannover; Christoph Zenzmaier, Innsbruck

\author{
Fälle-für-Alle ist ein berufsgruppeninternes Fallberichts- und Lernsystem für kritische \\ Ereignisse aus der geburtshilflichen Praxis für Hebammen. Mittels qualitativer Text- \\ analyse von 15 Interviews mit Hebammen aus Österreich, Deutschland und der Schweiz \\ wurde untersucht, welche Faktoren die Nutzung von Fälle-für-Alle fördern bzw. \\ hemmen können. Fälle-für-Alle ist ein wichtiger Beitrag zur Etablierung einer offenen \\ und konstruktiven Fehlerkultur.
}

Die Häufigkeit von unerwünschten Ereignissen (adverse events) in der Medizin liegt international zwischen 0,1-30\%, die Häufigkeit von Schäden zwischen 0,1$10 \%$ [1]. In der Geburtshilfe führen unerwünschte und potenziell vermeidbare Ereignisse teils zu schwerwiegenden und langwierigen Folgen für Mutter und/oder Kind. So wurden beispielsweise in England und Wales innerhalb eines Jahres über 62.000 geburtshilfliche Ereignisse an die National Patient Safety Agency gemeldet, von denen ca. 1/3 zu leichten bis moderaten Schäden führten und $2 \%$ mit schwerwiegenden oder tödlichen Folgen endeten [2]. In den vom National Health Service (NHS) betriebenen Einrichtungen wurden 2006/2007 im Median 144 Ereignisse pro 1.000 Geburten gemeldet [3].

Ein geeignetes Qualitätsmanagementsystem kann die Patientensicherheit deutlich erhöhen.

Beinahe die Hälfte der unerwünschten Ereignisse in der Medizin wären durch das Erfassen und Auswerten kritischer Ereignisse (critical incident) grundsätzlich vermeidbar und zwar durch den möglichen Lerneffekt zur Fehlervermeidung [4].

In den letzten 15 Jahren wurde im Gesundheitswesen in verschiedene Quali- tätssicherungsmaßnahmen investiert. Die meisten Institutionen haben mit der Einführung von Qualitätsmanagement auch Critical Incident Reporting Systeme (CIRS) implementiert. CIRS ist ein Berichtsystem, in dem ein „Zwischenfall ohne Schaden“ oder ein „kritisches Ereignis“ gemeldet werden kann. Es handelt sich hierbei in der Regel um „BeinaheSchäden“ oder „Beinahe-Unfälle“: Ereignisse mit potenziellem direktem Patientenschaden, die aber durch Interventionen oder Zufall nicht eingetreten sind. CIRS sind Frühwarnsysteme mit dem Ziel, durch Lernen aus Fehlern und Fehlerprävention das Risiko des Patienten zu minimieren [5].

Eine nationale Evaluationsstudie in den USA zeigte 2008, dass zwar fast alle Kliniken ein zentralisiertes Fehlermeldesystem eingeführt haben, es jedoch bei der Verbreitung unter den Angestellten und der Analyse von CIRS-Berichten große Lücken gab [6]. Bei einer Erhebung unter 831 Hebammen im deutschsprachigen Raum zeigte sich, dass nur 14\% der Hebammen unerwünschte Ereignisse im Rahmen von CIRS-Besprechungen aufarbeiten [7]. Fast 3/4 der befragten Hebammen gaben sogar an, dass an ihrer Institution kein CIRS verfügbar sei bzw. nicht zu wissen, ob es ein CIRS gibt.
Auch werden Fehlerberichte aus der Geburtshilfe möglicherweise zögerlicher in ein klinikinternes System gemeldet, da durch spezifische Fallkonstellationen (Geburt, Neugeborenes) eine Identifizierung der meldenden Abteilung leichter möglich ist, als beispielsweise eine Medikamentenverwechslung, die auf jeder beliebigen Station oder Funktionseinheit passiert sein kann. Solange keine konstruktive und wertschätzende Fehlerkultur installiert ist, führt dies potenziell zum subjektiven Gefühl von Scham oder Angst bei den Meldenden.

Neben dem unzureichenden Bekanntheitsgrad innerhalb der Kliniken besteht das Problem, dass mit einem klinikinternen CIRS kritische Ereignisse im außerklinischen/freiberuflichen Bereich nicht erfasst und analysiert werden können. Klinikinterne CIRS tragen somit im wesentlichen Tätigkeitsfeld von Hebammen nicht zu einer Fehlerprävention bei.

Deshalb entschieden sich Hebammen und Gutachterinnen im Herbst 2010, ein umfassendes freiwilliges Fallberichtsund Lernsystem für die gesamte Berufsgruppe der Hebammen für den deutschsprachigen Raum zu entwickeln [8,9]. Der dazu gegründete Verein Fälle-fürAlle e.V. stellt dabei das Lernen aus Fehlern in den Vordergrund, weshalb das 
entstandene Meldeportal Fälle-für-Alle als CIRLS (Critical Incident Reporting and Learning System) bezeichnet wird.

Eine vorhergehende Bedarfsanalyse zeigte das große Interesse der Hebammen an einem benutzerfreundlichen und anonymen Fallberichts- und Lernsystem [7]. Das Meldeportal wurde Anfang 2013 gestartet (https://hebammen.meldeportal. ch/) (Abb. 1).

Ziel der vorliegenden Studie war es Faktoren zu identifizieren, die die Nutzung des Fallberichts- und Lernsystem fördern und unterstützen oder behindern, um Fälle-für-Alle weiterentwickeln und verbessern zu können. Es sollte das Stimmungsbild unter Hebammen erfasst werden im Zusammenhang mit der Internetplattform Fälle-für-Alle und mit der Fehlerkultur im Allgemeinen.

\section{Methodik}

Für die Erfassung eines Stimmungsbildes sind persönliche Gespräche in Form von leitfadengestützten Interviews zweckmäßig.

Im Zeitraum zwischen Dezember 2013 bis März 2014 wurden in Einzelinter- views zwei deutsche, drei schweizerische und neun österreichische Hebammen jeweils in ihrem Land befragt. Die Akquise erfolgte nach pragmatischen Aspekten im Arbeitskontext der Forscherinnen in verschiedenen Settings und Sektoren. Um eine Atmosphäre des beruflichen Verstehens zu schaffen und um eine professionelle Distanz und Objektivität zu gewährleisten, wurden die Interviews von Hebammen geführt, die nicht an der gleichen Institution wie die Befragten tätig waren.

Der Interviewleitfaden beinhaltete Leitfragen zu den Themen CIRS Allgemein, die Internetplattform Fälle-für-Alle, gewünschte Ausbildungsinhalte, die Ansichten zur Fehlerkultur und das persönliche Erleben der Fehlerkultur in der Berufsausübung. Der Leitfaden ermöglichte den Interviewerinnen Wahlfreiheit hinsichtlich der Reihenfolge und der konkreten Formulierung der Fragen. Die Offenheit der Fragen ließ den befragten Hebammen Raum, eigene Anliegen und Gedanken zu äußern.

Die Interviews wurden aufgezeichnet, transkribiert und anonymisiert. Sie wurden mittels qualitativer Inhaltsanalyse ausgewertet.
Um alle Aussagen in ihren Zusammenhängen und Facetten berücksichtigen zu können, wurde die Software ATLAS.ti eingesetzt. Die Codierung erfolgte in den ersten Schritten der qualitativen Inhaltsanalyse offen, ohne von einer Theorie geleitet zu sein. Daraus entstanden im induktiven Vorgehen in der Analyse Kategorien welche über die durch die Interviewerfragen angestimmten Themen hinausgehen.

Im Folgenden werden einzelne Kategorien zusammengefasst dargestellt und mit entsprechenden Originalzitaten aus den geführten Interviews belegt.

\section{Ergebnisse}

\section{Nutzung und Bekanntheit}

Zum Zeitpunkt der Befragungen ist das Meldeportal Fälle-für-Alle seit ca. 8-12 Monaten online verfügbar. Die meisten der befragten Hebammen haben sich in der Vorbereitung auf das Interview mit dem Meldeportal vertraut gemacht. Teilweise war das Portal bereits vorher bekannt.

Die meisten Hebammen nutzen das Portal passiv durch Studium der aufbe- 
reiteten Fälle bzw. deren Einbeziehen in Teambesprechungen zu ähnlichen Ereignissen. Eine außerklinische/freiberufliche Hebamme gibt an, bereits einen Fall bei Fälle-für-Alle gemeldet zu haben. Die überwiegende Mehrheit will das Portal durch gezielte Mundpropaganda bekannter machen, was darauf schließen lässt, dass die Hebammen prinzipiell vom Nutzen eines berufsgruppenspezifischen Fallberichts- und Lernsystems überzeugt sind.

\section{Potenzieller Nutzen}

In den Interviews wird deutlich, welchen Nutzen sich die Hebammen von einem Fallberichts- und Lernsystem wie Fällefür-Alle erwarten. Eine zentrale Stellung nimmt hierbei das Lernen ein. So weisen einige Hebammen darauf hin, dass man mit Hilfe des Meldeportals aus Fehlern anderer lernen und auf potenzielle Fehler aufmerksam werden kann, wie die Aussage einer Klinikhebamme zeigt:

„Na, aber so war das. Ich hab reingeschaut und mir gedacht: Boah, das könnte ich sein, ja. Mir ist was Ähnliches ... es ist nichts passiert und das ist nur mir aufgefallen, aber das ist eine Fehlerquelle, die ... und dann hab ich mit anderen Kolleginnen darüber gesprochen.“

Des Weiteren geben die Hebammen an, dass die aufbereiteten Fälle helfen können, eigene Fehler einzuordnen. Eine leitende Klinikhebamme hebt die wissenschaftliche Behandlung der gemeldeten Fälle hervor und bezieht sich dabei vor allem auf die Literaturverweise im Meldeportal. Eine weitere Klinikhebamme meint, das Lesen von Fällen würde sich gut dazu eignen, Leerzeiten im Nachtdienst sinnvoll zu nutzen.
Einen Vorteil des Onlineportals gegenüber einem klinikinternen CIRS sehen einige Hebammen in einer höheren Anonymität:

„hmm, also ich hab jetzt kurz nachgedacht warum das bei uns nicht funktioniert mit den Fehlern, die man anonym eingeben kann weil, weil bei uns die (lacht) Anonymität ja einfach nicht klappt. Weil wenn da irgendein tragischer Zwischenfall war, weiß man einfach welche Hebamme dabei war. Also deswegen ... gibt's das nicht bei uns und ... insofern ist es super so etwas wie Fälle-für-Alle zu haben, weil da ... da kommen einfach mehr und da ist die Anonymität wirklich ... denke ich mal sicher"

Der Großteil der interviewten Hebammen fühlt sich durch Fälle-für-Alle unterstützt bzw. profitiert davon und bescheinigt dem Portal großes Wachstumspotenzial.
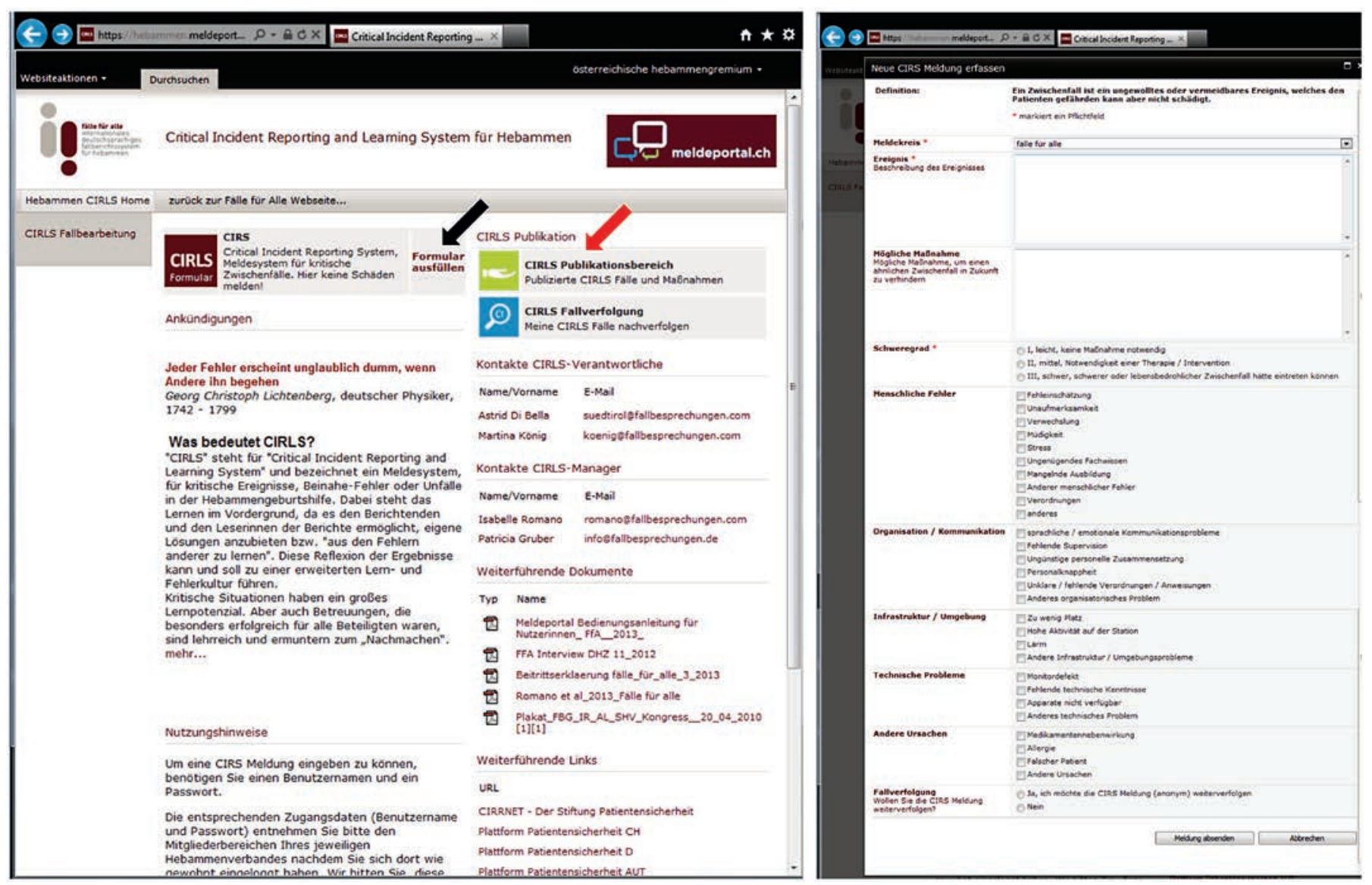

Abb. 1 Fälle-für-Alle Meldeportal.

Linke Seite: Screenshot der Startseite https://hebammen.meldeportal.ch/. Bei anklicken des Buttons „Formular ausfüllen“ (schwarzer Pfeil) erscheint das Meldeformular (Screenshot rechte Seite). Im CIRLS Publikationsbereich (roter Pfeil) sind die bearbeiteten Fälle einsehbar. 
Einige Hebammen betonen den aus ihrer Sicht großen Nutzen speziell für den außerklinischen/freiberuflichen Bereich. Eine Klinikhebamme fasst die Vorteile zusammen:

„...also ich sehe ein großes Potenzial überhaupt, dass man sich vernetzt als Hebammen ... dass gerade freiberufliche Hebammen, die keiner Institution angehören, sich grundsätzlich Gedanken darüber machen, über Qualitätsmanagement oder Standards, und sich eben auch mit anderen Hebammen absprechen. Nicht dass jede für sich selber schaut, und dass man eben auch aus Fehlern lernen kann und auch von anderen Sachen lernen kann. Einfach diese Vernetzung das Bewusstsein dafür, dass sich jede Hebamme Gedanken dazu macht.“

\section{Was hemmt die Nutzung?}

Trotz der oben genannten Vorteile werden in den geführten Interviews auch hemmende Faktoren für die Nutzung deutlich. So ist eine leitende Klinikhebamme beispielsweise unsicher, ob die Anonymisierung gewährleistet ist. Andere Hebammen empfinden das Thematisieren von Fehlern in der Geburtshilfe generell als unangenehm und verängstigend, was prinzipiell die Nutzung von CIRS hemmt:

„Also freiwillig würde ich wahrscheinlich nicht drauf gucken, weil es mir Angst macht. Ich merke das immer in Fortbildungen oder bei Vorträgen. Wenn es zu solchen Themen kommt, habe ich immer ganz, ganz große Angst, ich kriege wirklich schwitzige Hände, Schweißausbrüche und bin froh, wenn der Teil des Vortrages vorbei ist und halte es so schon schwer aus. Ich glaube, auf solche Portale würde ich mich freiwillig nicht begeben."

Auch eine allgemeine, nicht konkret begründete Hemmschwelle wird thematisiert:

„Ich hab auch schon überlegt, das in das „Fälle-für-Alle“ reinzugeben, aber hatte irgendwie ... Es ist trotzdem eine Hemmung da.“

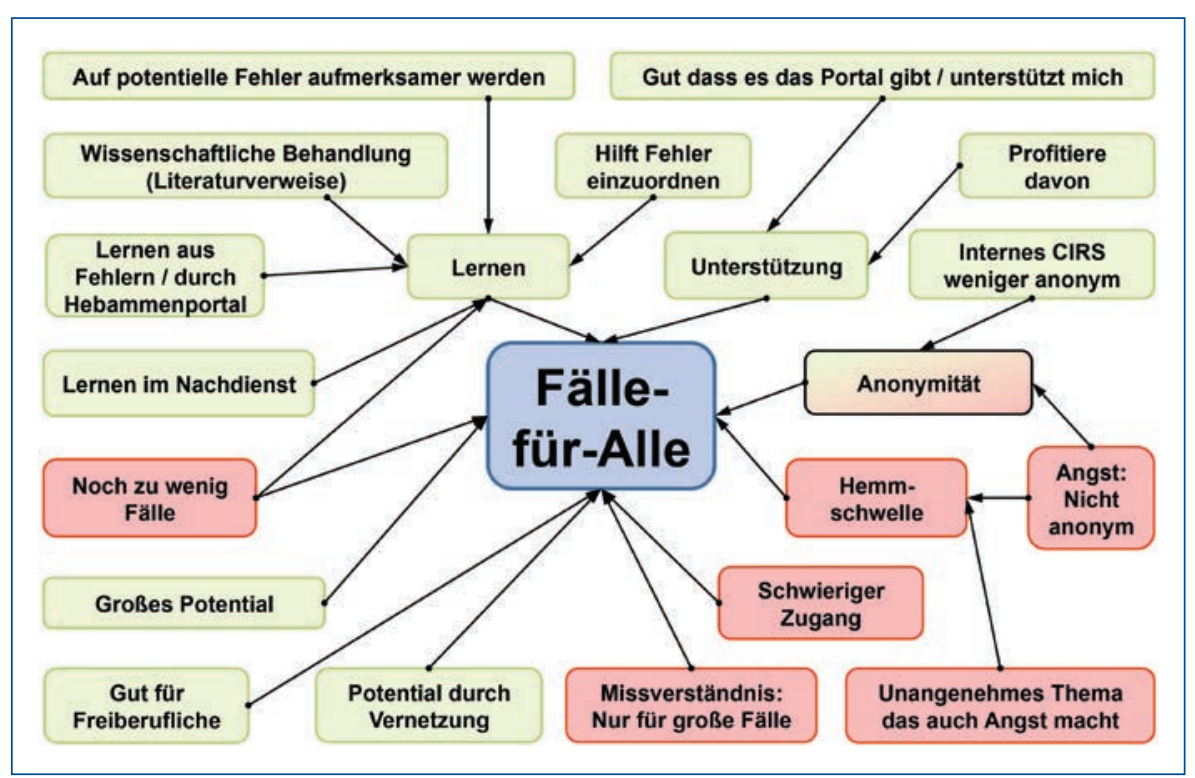

Abb. 2 Förderliche und hemmende Faktoren.

Netzwerkdarstellung der qualitativen Inhaltsanalyse zu förderlichen und hemmenden Faktoren der Nutzung von Fälle-für-Alle.

Einige Hebammen berichten von technischen Schwierigkeiten bzw. Zugangsschwierigkeiten beim Online-Meldeportal. Auch die Tatsache, dass zum Zeitpunkt der Interviews noch wenige Fälle im Portal aufbereitet waren, beschränkt den Nutzen als Lernportal.

In den Interviews zeigt sich eine Unsicherheit darüber, was ein meldewürdiges Ereignis ist. Es fällt offensichtlich schwer, kritische Ereignisse richtig einzuordnen. Vor allem Beinahe-Schäden im eigenen Bereich scheinen bagatellisiert zu werden oder als zu gering für eine Meldung erachtet. Als Ereignis werden teilweise nur Fehler ab einer gewissen (potenziellen) Schadensgröße wahrgenommen:

„also mit Fehler assoziiere ich immer ... ja wirklich was Arges, grad in der Geburtshilfe also jetzt nicht eine Kleinigkeit" leitende Klinikhebamme; „den Katheter, ah genau den werde ich hinein schreiben ... ... ich hab gedacht immer wenn nur was ganz schlimmes irgendwie passiert, ach ja stimmt, ja ich schreib den Katheter rein“ - Klinikhebamme; „...wenn ich ein kritisches Ereignis erlebt hätte ... ja dann hätte ich es vielleicht gemeldet." (freiberufliche Beleghebamme)
Förderliche und hemmende Faktoren für die Nutzung von Fälle-für-Alle sind in Abb. 2 zusammengefasst.

\section{Konstruktive Fehlerkultur}

Für das Fallberichts- und Lernsystem Fälle-für-Alle gelten die gleichen Prinzipien wie für alle CIRS/CIRLS.

\section{Zu den essenziellen Grundprinzipien} gehört neben Anonymität, Vertraulichkeit und Sanktionsfreiheit auch die Freiwilligkeit.

Ausschlaggebend für den Erfolg ist dabei eine entsprechende Fehler- und FeedbackKultur. Dies bezieht sich insbesondere auf einen wertschätzenden Umgang mit sich selbst und den Kolleginnen bei der kritischen Reflexion der eigenen Praxis. Das Lernen aus Fehlern stellt die fruchtbarste aller theoretisch möglichen Fehlerumgangsformen dar, da Fehlerlernprozesse die Wahrscheinlichkeit der Fehlerwiederholung in der Zukunft senken [10].

Diese wird im Gesundheitswesen nur teilweise gelebt. Eine stark hierarchisch geprägte Struktur fördert das Misstrauen zwischen den Berufsgruppen und 
führt zu Kommunikationsbarrieren zwischen den hierarchischen Ebenen.

„und wenn's die Chefin nicht erfährt, dann braucht man es auch mit ihr nicht (lacht) ausreden, also, immer Glückssache." (Klinikhebamme)

Auch ist es noch immer weit verbreitete Praxis, bei Auftreten eines Fehlers sofort nach einem Schuldigen zu fragen, anstatt mithilfe einer Fehlermeldung die oftmals im System begründete Fehlerursache zu ermitteln. Dieser Mangel an offener Fehlerkultur verringert die Motivation, sich an freiwilligen Berichtsystemen zu beteiligen.

„In ... diesem Thema Schuld, Konflikt Arzt-Hebamme, hat auch einen sehr historischen Hintergrund, diese Schuldzuweisungen“ - Leitende Klinikhebamme; „... wann immer ein Fehler passiert in unserer Gesellschaft, wird immer nach dem Schuldigen gesucht. Und solange diese Schuldfrage so vorherrschend ist und in der Gesellschaft, ja, keine Fehlerkultur gelebt werden kann, sondern immer gleich sehr hart durchgegriffen wird, finde ich diese Frage sehr schwierig zu beantworten (...) Die Erfahrungen als Klinikhebamme haben gezeigt, dass es, ja, je nach Umfeld möglichst darauf bedacht wird, dass man eben nicht darüber spricht. In der einen Klinik wurden Fehler möglichst totgeschwiegen. In der anderen Klinik war eine gute Fehlerkultur (...)“- Klinikhebamme

Daher ist es in den deutschsprachigen Ländern Deutschland, Österreich und Schweiz offenbar notwendig, die Beschäftigten im Gesundheitswesen zu schulen und mit einer konstruktiven Fehlerkultur und Fehlermanagementmethoden vertraut zu machen. Die überwiegende Mehrzahl der befragten Hebammen würde solche Lehrinhalte idealerweise in der Grundausbildung verankern, teilweise bereits am Anfang der Hebammenausbildung oder gegen Ende des Bachelorstudiums. Zusätzlich sollten vertiefende Fortbildungen angeboten werden. Die meisten Hebammen plädieren dafür, Bildungsmaßnahmen zur Fehlerkultur wiederkehrend anzu- bieten, z.B. das Wissen zusätzlich zur Grundausbildung in Masterstudiengängen zu erneuern und zu vertiefen. Darüber hinaus sei auch das Erleben einer offenen Fehlerkultur im Berufsalltag nötig:

„Ich denke, eine Fehlerkultur muss wirklich, muss man auch leben, im Alltag leben und im Alltag erlernen und das geht nur, wenn du mitten drin steckst und wenn es darum geht, unter den Geburten oder nach den Geburten sich gegenseitig die Schuld zuzuschieben und eben ... Ja, man muss es quasi in der ... Man muss es in der Praxis üben und erfahren.“

Problematisch ist dabei vor allem die Umsetzung des gelernten Verhaltens in die Praxis, da die Hebammen in der Ausbildung in einem Umfeld ohne gelebte
Fehlerkultur in eine angreif- und verletzbare Position geraten könnten:

„Das würde ich nur anbieten wollen, wenn ich wüsste, dass das Krankenhaus so eine Fehlerkultur auch lebt. Es bringt nichts, eine Schülerin in einer Fehlerkultur auszubilden, wenn sie aber das versucht, in der Klinik zu leben und dann ins offene Messer läuft. Das geht nur, wenn alle Beteiligten auch ernsthaft an einer Fehlerkultur interessiert sind und ich die Schülerinnen dadurch nicht verletzlich oder verletzbar mache."(Klinikhebamme)

Anonyme Instrumente wie Fälle-für-Alle ermöglichen es, eine konstruktive Fehlerkultur zu leben ohne sich solchen Gefahren auszusetzen, und können so helfen, eine offene Fehlerkultur zu erlernen:

\section{Wie erhalte ich Zugang zu Fälle-für-Alle?}

Fälle-für-Alle ist ein berufsgruppeninternes, elektronisches Online-Meldesystem für Erfahrungsfälle aus der geburtshilflichen Praxis für Hebammen.

Zugang zum Meldeportal erhalten alle Hebammen, die bei einem der unten genannten Hebammenverbände Mitglied sind. Die Zugangsdaten sind im Mitglieder- oder Servicebereich der einzelnen Verbände ersichtlich.

Die Verwendung der Zugangsdaten eines Verbandes garantieren die Anonymität des Melders, da diese Zugangsdaten von allen Mitgliedern des Verbandes verwendet werden und nicht personalisiert sind.

Alternativ können Hebammen durch Mitgliedschaft beim Verein „Fälle-für-Alle e.V.“ Zugang zum Portal erhalten (nähere Informationen auf der Internetseite des Vereins).

\section{Wo finde ich Fälle-für-Alle?}

Das Meldeportal Fälle-für-Alle:

https://hebammen.meldeportal.ch/

Informationen über den Verein / Mitgliedschaft:

http://www.fälle-für-alle.org/

Zugang haben Mitglieder des Vereins „Fälle-für-Alle e.V.“ und folgender

Hebammenverbände:

- Deutscher Hebammenbund (DHV)

- Bund freiberuflicher Hebammen Deutschlands (BfHD)

- Schweizerischer Hebammenbund (SHV)

- Österreichisches Hebammengremium (ÖHG)

- Hebammenkollegium Bozen

\section{Was soll gemeldet werden?}

Bitte keine Schadensfälle. Ansonsten gilt: Jedes Ereignis zählt! 
„Dass das zu uns gehört, dass man sich reflektiert und eben diese Meldesysteme nutzt und halt wirklich so, dass es jedem passieren kann im Arbeitsalltag." (Klinikhebamme)

\section{Diskussion}

Obwohl der Umgang mit CIRS im Klinikalltag nicht flächendeckend implementiert ist [7], zeigt sich in den Interviews, dass die Hebammen einem Fallberichtsund Lernsystem prinzipiell positiv gegenüberstehen. Sie fühlen sich durch ein solches System in ihrer Arbeit unterstützt und sehen die Vorteile vor allem in der Funktion als Lernplattform.

Wie bereits in der vorhergehenden quantitativen Befragung [7] zeigte sich ein besonderer Bedarf für den außerklinischen/ freiberuflichen Bereich, da hier klinikinterne Qualitätsmanagementsysteme wie CIRS nicht greifen. Ähnliches gilt für niedergelassene Ärzte, für die aus diesem Grund in den letzten Jahren CIRLS online implementiert wurden (z.B. www.jederfehler-zaehlt.de oder CIRSmedical).

Zu den Faktoren, die die Nutzung von Fälle-für-Alle hemmen können, zählen Zweifel, ob ein solches System wirklich die Anonymität wahren kann, und technische Schwierigkeiten beim Zugang zum Meldeportal. Vertraulichkeit und Anonymität sind Grundprinzipien eines CIRLS und gerade eine Online-Plattform hat den Vorteil einer systeminduzierten Anonymität des Melders [4]. Die Anmeldung und Erfassung erfolgt nicht mit persönlichen Daten, sondern über einen einheitlichen generischen Benutzer. Somit bleibt der Verfasser der Meldung völlig anonym. Das Risiko eines inhaltlichen Wiedererkennens im persönlichen Berufsumfeld wird durch die länderübergreifende Verbreitung des Onlineportals und die damit auch verbundene hohe Zahl an Meldungen minimiert.

Die Hemmschwelle, Ereignisse zu melden, bzw. der stark emotionale Umgang mit dem Thema deuten darauf hin, dass eine offene und konstruktive Fehlerkultur im Gesundheitswesen leider nicht flächendeckend etabliert ist. Gerade die Nutzung eines Fallberichts- und Lernsystems in einem geschützten Bereich wie Fälle-für-Alle (Online-System, unabhängig von Kliniken und ohne Meldung an Vorgesetzte; berufsgruppeninterne Nutzung) kann dazu beitragen, Erfahrungen im richtigen Umgang mit kritischen Ereignissen zu sammeln, Sicherheit im Fehlermanagement zu erlangen und eine offene Fehlerkultur zu entwickeln.

Die befragten Hebammen waren sich teilweise nicht sicher, welche Ereignisse an Fälle-für-Alle gemeldet werden sollten. Wichtig und richtig ist, dass an ein CIRS/CIRLS nur kritische Ereignisse und keine Schadensfälle gemeldet werden sollen. Es sollen nur Situationen geschildert werden, die „nochmals gut gegangen sind" und nicht offensichtlich eingetretene Schäden. Ansonsten gibt es keine

\section{Einschränkungen.}

Jedes Ereignis, sollte es auch noch so unbedeutend erscheinen, kann dazu beitragen, einen Fehler an anderer Stelle zu verhindern.

Hebammen sind außerdem eingeladen, neben kritischen Ereignissen und Beinahe-Fehlern, Best-Practice-Fälle aus der täglichen Arbeit einzugeben. Mit jedem aufbereiteten Fall steigt der Wert von Fälle-für-Alle als Lernplattform und trägt schließlich im Interesse der zukünftigen Mütter und deren Kinder dazu bei, das Gesundheitssystem weiter zu verbessern.

\section{Literatur}

1 Schrappe M, Lessing C. Zur Häufigkeit von Medizinschadensfällen. In: Madea B, Barth S, Hrsg. Medizinschadensfälle und Patientensicherheit. Köln: Dt. ÄrzteVerlag; 2007: 21-31

2 O'Neill O. Safe Births: Everybody‘s Business: An Independent Inquiry into the Safety of Maternity Services in England. London: King's Fund; 2008

3 Healthcare Commission. Towards better births: a review of maternity services in England. London: Commission for Healthcare Audit and Inspection; 2008
4 Kaufmann M, Staender S, Scheiddegger D. Meldesysteme, Beispiel CIRS. In: Holzer E, Hrsg. Patientensicherheit: Leitfaden für den Umgang mit Risiken im Gesundheitswesen. Wien: Facultas; 2005: 155-158

5 Schrappe M. Das sichere Krankenhaus. In: Holzer E, Hrsg. Patientensicherheit: Leitfaden für den Umgang mit Risiken im Gesundheitswesen. Wien: Facultas; 2005: 56-61

6 Farley DO, Haviland A, Champagne S et al. Adverse-event-reporting practices by US hospitals: results of a national survey.

7 Romano I, König M, Ederer C et al. Fälle-für-Alle e.V.: Welche Erwartungen haben Hebammen an ein Fallberichtssystem? Hebamme; DOI: 10.1055/s-0032-1331044

8 Gruber P, Schwarz C. Fälle-für-Alle oder: „Man muss nicht jeden Fehler selber machen!" Hebamme; DOI: 10.1055/s-0030-1267813

9 König M. „Fälle für Alle“ - Ein neues Fallberichts- und Lernsystem für Hebammen. Österreichische Hebammenzeitung 2012; 01: 25-27

10 Lobnig H, Nowak P, Pelikan JM (1999): Die Umsetzung der Vision des Gesundheitsfördernden Krankenhauses. In: Pelikan JM, Wolff S (Hg.): Das gesundheitsfördernde Krankenhaus. Weinheim, München: Juventa, (S. 51-66)

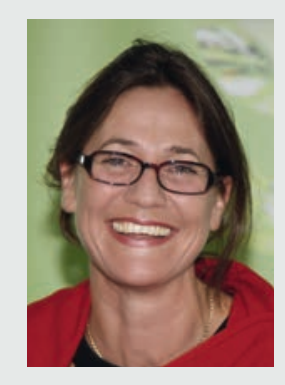

Martina König-Bachmann, MHPE Studiengangsleiterin FH-Bachelor-Studiengang Hebamme Lehrgang Master of Science in Advanced Practice Midwifery fhg-Zentrum für Gesundheitsberufe Tirol GmbH Innrain 98 A-6020 Innsbruck E-Mail: martina.koenig-bachmann@ fhg-tirol.ac.at 